\title{
Energy Efficient Routing Strategies for Large Scale Wireless Sensor in Heterogeneous Networks
}

\author{
0. Sampath1, Dr. P. Suryanarayana Babu² \\ ${ }^{1}$ Research Scholar, ${ }^{2}$ Research Supervisor \\ Rayalaseema University, Kurnool, Andhra Pradesh, India
}

\begin{abstract}
The efficiency of a wireless sensor network depends on its life time. By conserving the energy of each sensor for increase in the network life time. The basic operations of a wireless sensor network are sensing [1] the data to the energy sink term is for next transmitting node. The communication or routing [2] process operation be allowed in any operations for all nodes [3]. We propose to select a specific collection of nodes for communication with considering the importance of wireless sensor where security [4] and power usage [5] is taken as top priority.
\end{abstract}

KEYWORDS: Transmitting node [6], neighboring node [7], energy sink [8].

\section{INTRODUCTION}

Wireless sensor networks (WSNs) have received tremendous attention in recent years because of the development of sensor devices, as well as wireless communication technologies. It is usually randomly deployed in inaccessible terrains, disaster areas, or polluted environments, where battery replacement or recharge is difficult or even impossible to be performed. For this reason, network lifetime is of crucial importance to a WSN.

In a WSN, sensor nodes are typically operated by batteries, which are limited in energy capacity, and difficult or even impossible to be replaced or recharged. For this reason, power control is needed to efficiently make use of the limited energy resources in order to minimize the energy consumed by the sensor nodes and thus prolong network lifetime. For this purpose, energy efficiency must be considered in every aspect of network design and operation, not only for individual sensor nodes, but also for the communication of the entire network.

Recent advances in micro-electro-mechanical systems (MEMS) technology have made the deployment of wireless sensor nodes a reality [1, 2], in part, because they are small, inexpensive and energy efficient. Each node of a sensor network consists of three basic subsystems: a sensor subsystem to monitor local environmental parameters, a processing subsystem to give computation support to the node, and a communication subsystem to provide wireless communications to exchange information with neighboring nodes.

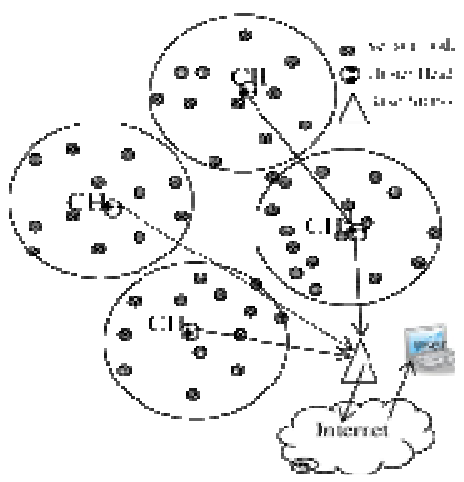

Fig.1: A Two tier- Hierarchical -four cluster based distributed wireless sensors
First, due to the relatively large number of sensor nodes, it is not possible to build a global addressing scheme. Thus, traditional IP-based protocols are not recommended for WSN use. Furthermore, sensor nodes that are deployed in an ad-hoc manner need to be self-organizing as the ad-hoc deployment of these nodes requires the system to establish connections and cope with the resultant nodal distribution, Flooding is a simple technique that can be used to broadcast information in wireless sensor networks, however it requires significant resources because each node receiving a message must rebroadcast it.

Hierarchical protocols are based on clusters because clusters can contribute to more scalable behavior as the number of nodes increases, provide improved robustness, and facilitate more efficient resource utilization for many distributed sensor coordination tasks.

Low-Energy Adaptive Clustering Hierarchy

(LEACH) is a cluster-based protocol that minimizes energy dissipation in sensor networks by randomly selecting sensor nodes as cluster heads.

The rest of the paper is organized as follows: Section II references the related work. Section III presents the preliminaries. Section IV Proposes. Section V does the analysis. Section VI shows the simulation results. And conclusion is in Section VII.

\section{RELATED WORK}

Routing is the most vital and energy consuming task in wireless sensor networks (WSNs). The two foremost prospects of a WSN are lower hardware cost and constant energy drainage. Though heterogeneity aims to achieve the former, homogeneity assumes the persistent drainage of energy. Both characteristics are anticipated to be integrated within the same network. The objective of this paper is to provide the comparative analysis of homogeneous vs. heterogeneous networks along with the cost analysis to decide the energy-hardware trade off.

A node structure as shown in figure 2 typically consist of four basic components: a sensing unit, a processing unit, a communication unit, and a power unit [5]. 

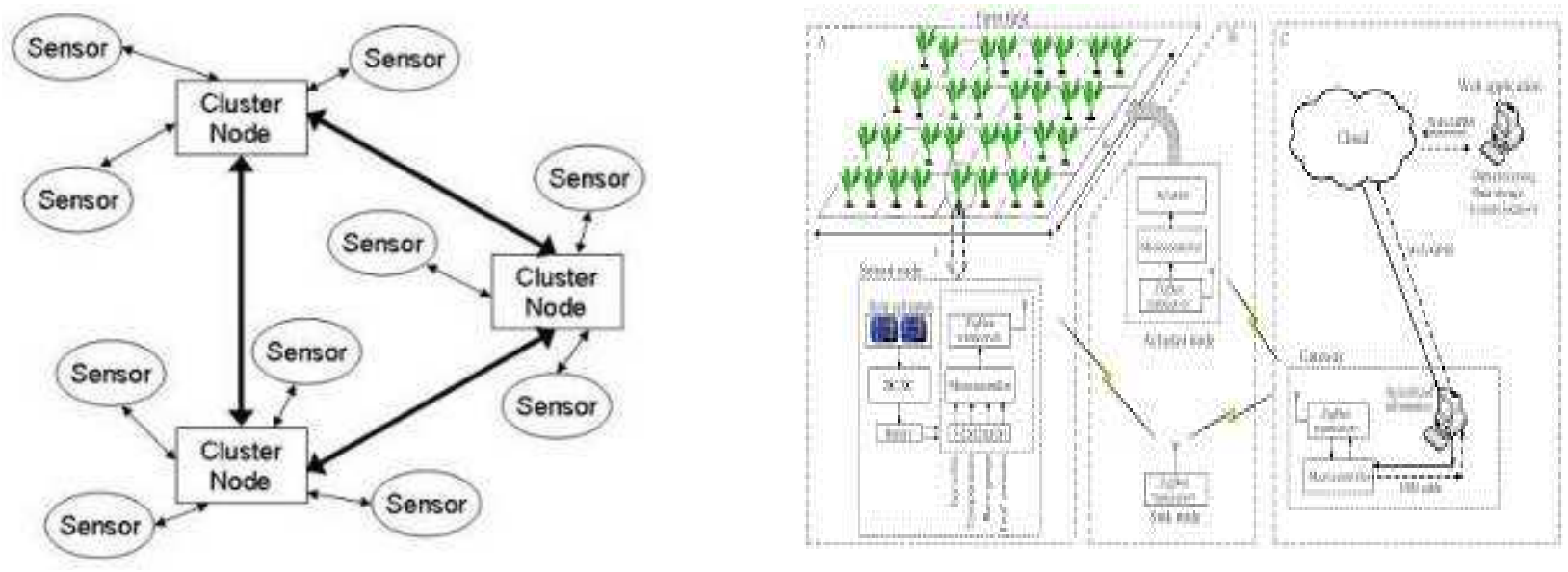

Fig-2: Cluster Node Bi-Directional Communication.

\section{PRESENT AND PRELIMINARY WORK:}

As per the basic steps we have taken, we will discuss each in implementation way:

1. Deploying the nodes in network with global addressing.

In an communication network where we have a wireless devices there is more chance of getting the data sharing over an number of devices. We consider the aspect of data sharing as main goal in wireless devices from every node to another node but with registered id (R-NID) in the network.

Whenever a node is ready to communicate to the available registered device we first make that node to register in the network with basic information and generate an ID for the new node, after getting id that is termed in network global addressing. The network table is updated and repeated communication to this node is then moved as cluster (where each cluster has specific attribute grouped together).

Algorithm with technical orders:

1. We have $\mathrm{n} 1, \mathrm{n} 2, \mathrm{n} 3$ and $\mathrm{n} 4$ nodes already present in the network.

\begin{tabular}{|c|c|c|}
\hline $\begin{array}{l}\text { Host IP: considering the peer-peer } \\
\text { connection for all registered nodes }\end{array}$ & \multirow{2}{*}{$\begin{array}{l}\text { Rules for register id: } \\
\text { Register Id: Considering the active } \\
\text { or inactive communication mode } \\
\text { with in network }\end{array}$} & $\begin{array}{l}\text { R-NID:node1: } \\
\text { host1/peer1/node1 }\end{array}$ \\
\hline $\begin{array}{l}\text { Subnet mask address } \\
\text { (node1 of network1:1) }\end{array}$ & & $\begin{array}{l}\text { R-NID:node1: } \\
\text { host1/peer1/node1:1 }\end{array}$ \\
\hline $\begin{array}{l}\text { Subnet mask address } \\
\text { (node2 of network1:2) }\end{array}$ & \multirow{2}{*}{$\begin{array}{l}\text { Register Id: Considering the } \\
\text { generated id on new node } \\
\text { communication mode with in } \\
\text { network }\end{array}$} & $\begin{array}{l}\text { R-NID:node1: } \\
\text { host1/peer1/node1:2 }\end{array}$ \\
\hline $\begin{array}{l}\text { Subnet mask address } \\
\text { (node3 of network1:3) }\end{array}$ & & $\begin{array}{l}\text { R-NID:node1: } \\
\text { host1/peer1/node1:3 }\end{array}$ \\
\hline $\begin{array}{l}\text { Subnet mask address } \\
\text { (node4 of network1:4) }\end{array}$ & $\begin{array}{l}\text { Register Id: Considering the cluster } \\
\text { of peer mode with in network }\end{array}$ & $\begin{array}{l}\text { R-NID:node1: } \\
\text { host1/peer1/node1:4 }\end{array}$ \\
\hline
\end{tabular}

2. Connecting to sensors when data communication is needed.

The authentic node termed till now is only as global addressing. But the node is not fully ready to get data transfer.so, an sensor will be set for each device with global addressing.suppose we have three node and 1 sensor then the active node termed and ready for communication are assigned to sensor data transfer mode: the switch in and the switch off action are internally termed for sensor to start data transfer with two parameters (one is sender global address and other is receiver global address).

After the communication is over an signal from the receiver is sent to deactivate the connection (basically and unsyc signal sent) .Further if any node is ready to send data the the sensor is connected with the node(basically with syn signal).

In implementation we take 2 sensors (accessiblility to nodes having R-NID

\begin{tabular}{|c|c|}
\hline $\begin{array}{l}\text { Sensor data: considering the peer-peer connection for all active } \\
\text { node }\end{array}$ & \multirow{3}{*}{$\begin{array}{l}\text { Dedicated channel of communication for } \\
\text { allocated sensors }(1,2) \text { set to SYN }\end{array}$} \\
\hline $\begin{array}{l}\text { Sensor data: SET to active or } 1 \text { (node1 of with other registered } \\
\text { node }(2,3,4) \text { communication with sensor } 1 \text { ) }\end{array}$ & \\
\hline $\begin{array}{l}\text { Sensor data: SET to active or } 1 \text { (node } 2 \text { of with other registered } \\
\text { node }(3,4) \text { communication with sensor } 2 \text { ) }\end{array}$ & \\
\hline $\begin{array}{l}\text { Unavailable mode for sensors when nodes what to communicate: } \\
\text { Case1: Node1 (allotted to } 2,3,4 \text { ) and node } 3 \text { is requesting for } \\
\text { communication } \\
\text { Case2: Node2 (allotted to } 3,4 \text { ) and node } 3 \text { is requesting for } \\
\text { communication }\end{array}$ & $\begin{array}{l}\text { Nodes in network are in wait state set to } \\
\text { UNSYN }\end{array}$ \\
\hline
\end{tabular}


3. After each node communication, updating the node allocation table.

Each node data transfer will be repeatedly get done with updation of nodes strategy as a table in network. The attributes get updated are(node no,R-NID,global address,SID/RID,flag).

\begin{tabular}{|c|c|c|c|c|}
\hline Node no & R-NID & Global address & SID/RID & flag \\
\hline $\begin{array}{l}\text { Each node } \\
\text { number } \\
\text { with } \\
\text { (n1,n2,n3 } \\
\text {...nk) }\end{array}$ & $\begin{array}{l}\text { Each with } 16 \text {-bit of short } \\
\text { address and prefixed with } \\
\text { an level of communication } \\
(01,02,03 \& 04)\end{array}$ & $\begin{array}{l}\text { Each of 16-bit } \\
\text { address and } \\
\text { hierarchically }\end{array}$ & $\begin{array}{l}\text { Alphanumber } \\
\text { sender and receiver } \\
\text { id with general } \\
\text { rules on framing the } \\
\text { length with } 63 \text {. }\end{array}$ & $\begin{array}{l}\text { Flags are } \\
01 \text { for new node registered, } \\
02 \text { for already registered but } \\
\text { not sensor used, } \\
03 \text { for sensor used for already } \\
\text { established connection and o } 4 \\
\text { for new sensor and new node } \\
\text { communication }\end{array}$ \\
\hline
\end{tabular}

The R-NID is assigned a 16 bit short address, which is unique within a WPAN or SMAG domain, and remains fixed irrespective of its location within the WPAN. All three levels of addresses are created hierarchically.16 bits short addresses are assigned to a R-NID at the time of deployment.

4. Sensors deactivation after no path for communication (for efficient energy consumption)

Node with global addressing and frame of data (datatype, data stream, length, receiver address) are updated in communication path as sensor request is given by each node as SYNC and UNSYNC. The method of allocation depends on the priority and with security terms checked in each access and request. Basic security terms and priority scheduling is used and then establishes the connection with GRANT SYNC signal from each node.

Basic data frame as follows:

\begin{tabular}{|c|c|c|c|}
\hline Data type & Data stream & Length & $\begin{array}{c}\text { Receiver } \\
\text { address }\end{array}$ \\
\hline $\begin{array}{c}\text { Fixed data type is recommended } \\
\text { to get rules for security issues }\end{array}$ & $\begin{array}{c}\text { Data type decides the stream } \\
\text { to be in uni or bi directional }\end{array}$ & $\begin{array}{c}\text { Length is fixed to } \\
\text { 16-bit data }\end{array}$ & 16-bit \\
\hline
\end{tabular}

After each communication the data end of node establishment is decided by signal-SYNC and GRANT SYNC for every UNSYNC signal.

5. Listing all the active nodes and sensors in network.

The node communication is updated in table with sensor user and active nodes using them.

(Node id, sensor id, receiver id, sensor id) the collection of information is repeatedly updated and if any sensor is damaged then the data is resend to the sender with an flag signal set to nonzero value. This non-zero value is always generated when the sensor is in active(damaged, no signal, not working or any other technical problems).

The collection of node is termed as Cluster by following rules:

1. Repeatedly two registered nodes communicating to each other.

Registration process:

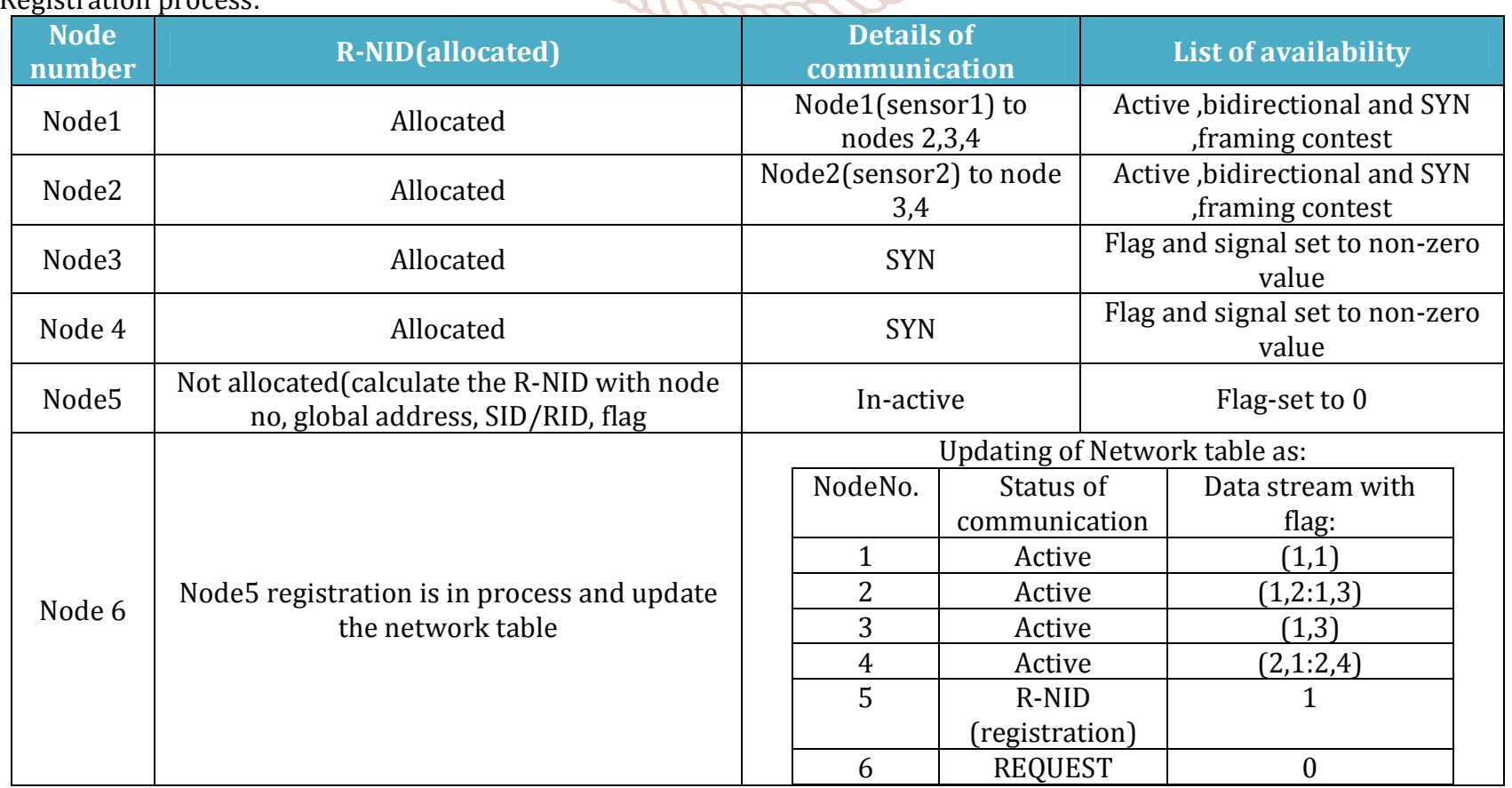


2. Two or more registered nodes sending or receiving the same type of data frames. Data frames allocation strategy:

We have 6 nodes (n1...n6) in our network with two sensors(s1 and s2):

\begin{tabular}{|c|c|c|c|}
\hline Frame & Data stream & Request status & Sensor \\
\hline Data1:frame & Data1:frame & SYN:write-Node & Node1:sensor \\
1:write mode & 1:block1:1,2 & 1 to Node2,3,4 & 1(SYN,BI-directional) \\
\hline Data2:frame & Data1:frame & SYN:read-Node & Node2:sensor \\
1:write mode & 1:block1:1,3 & 2 to Node 3,4 & 2(SYN,uni-directional) \\
\hline
\end{tabular}

Our problem revolves around the classification of nodes as normal ('Nn'), advanced ('Na') and super ('Ns') nodes for the simulation of Multi-MAF for heterogeneous network.

Assumption: Each node has same communication and sensing model.

To obtain the cost analysis of the heterogeneity. The Energy cost of a sensor node $=a+\beta E$

where ' $\alpha$ ' is the hardware cost, ' $\beta$ ' is the constant and ' $E$ ' is the battery energy of the normal node.

$E k={ }_{n} n=1 k(a k+\beta E k)$ where ' $k$ ' denotes the selected heterogeneity levels respectively. ' $E k$ ' denotes the different selected energy level of nodes correspondingly.

Assumption: Battery costs are not included in the hardware cost.

Most of the protocols designed for WSNs assume that the sensors have the same capabilities in terms of storage, processing, sensing, and communication. The resulting network is said to be homogeneous. In these types of networks, a pair of sensors would have the same lifetime if they have the same energy consumption rate. Some sensing applications, however, use sensors with different capabilities and accordingly the resulting network is said to be heterogeneous.

In the real world, the assumption of homogeneous sensors may not be practical because sensing applications may require heterogeneous sensors in terms of their sensing and communication capabilities in order to enhance network reliability and extend network lifetime [2]

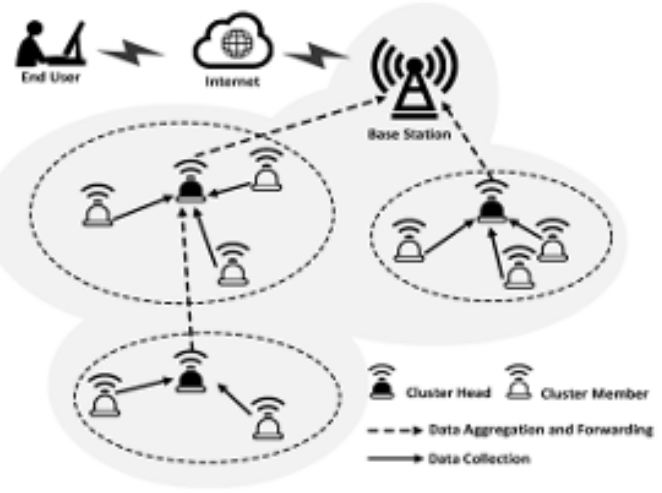

An HWSN can be represented by a directed graph $G=\{\mathrm{V}, \mathrm{E}\}$, where $V$ is the set of sensors (also called nodes), and $E$ is the set of links (also called edges) in the network. For example, if sensor $B$ is in the transmission range of sensor $A$, then there is a directed link from A to B. We assume graph G generated from the HWSN is a strongly-connected directed graph. Therefore, the HWSN is also strongly-connected.
We categorize the neighbor relationships of sensors into four categories: (1) In-out-neighbor; (2) In- neighbor; (3) Outneighbor; and (4) Non-neighbor. For two nodes A and B, as shown in Fig. 1, if $A \rightarrow B$ and $B \rightarrow A$, then $A$ and $B$ are Inout-neighbors of each other. If only $A \rightarrow B($ or $B \rightarrow A$ ), then $A$ (or $B$ ) is the In-neighbor of $B($ or $A$ ), and $B$ (or $A$ ) is the Outneighbor of $A$ (or $B$ ). If neither $A \rightarrow B$ nor $B \rightarrow A$, they are non-neighbors of each other.

We assume data is transmitted through lossy links. The packet loss rate of a link uv is defined as 1 minus the ratio of the number of packets $\mathrm{Nd}$ which are successfully received by node $v$ to the total number of packets Ns sent by $u$. That is, Plossrate $=1-\mathrm{Nd} / \mathrm{Ns}(1)$

The performance analysis of these basic strategies is evaluated using simulation derived for the following performance metrics:

Route discovery time (Latency): is the time the sink must wait before actually receiving the first data packet.

Average end-to-end delay of data packets: includes all possible delays caused by queuing, retransmission delays at the MAC and propagation and transfer times.

Packet delivery ratio: is the ratio of the number of data packets delivered to the destination and the number of data packets sent by the sender. Data packets may be dropped en route for several reasons:

e. $\mathrm{g}$. the next hop link is broken when the data packet is ready to be transmitted or one or more collisions have occurred.

\section{RESEARCH WORK: \\ Heterogeneous impact on the wireless sensor networks}

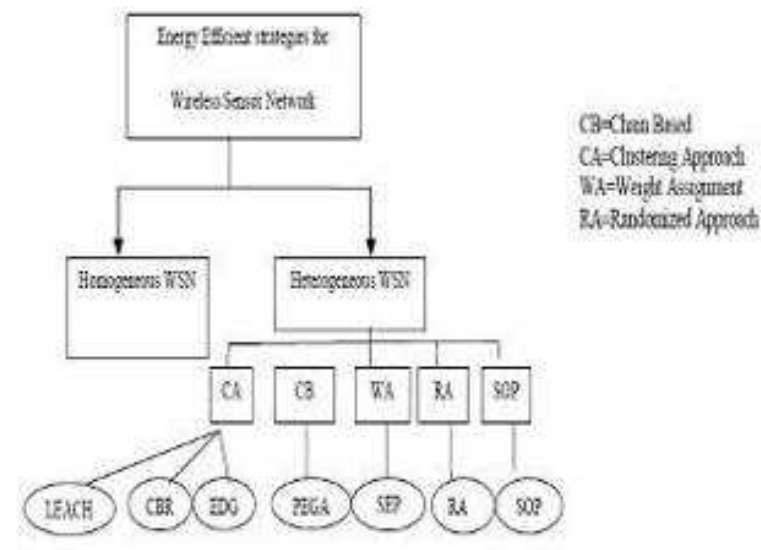

Placing few heterogeneous nodes in the sensor network can bring following three main benefits: 


\section{Prolonging network lifetime.}

In the heterogeneous wireless sensor network, the average energy consumption for forwarding a packet from the normal nodes to the sink in heterogeneous sensor networks will be much less than the energy consumed in homogeneous sensor networks.

Sensor nodes sense their environment, collect sensed data and transmit it to the BS. However, they are limited in power, computational capacity and memory. Placing few heterogeneous nodes in wireless sensor network is an effective way to increase network lifetime and reliability.

\section{Cluster based approach:}

In a hierarchical network, sensor nodes are organized into clusters, where the cluster members send their data to the cluster heads while the cluster heads serve as relays for transmitting the data to the sink. A node with lower energy can be used to perform the sensing task and send the sensed data to its cluster head at short distance.

This process can not only reduce the energy consumption for communication, but also balance traffic load and improve scalability when the network size grows.

Depending on the objective and the methodology, numerous clustering algorithms have been proposed. The complexity and convergence rate of these algorithms can be constant or dependent on the number of CHs and/or sensors.

Low-energy adaptive clustering hierarchy (LEACH) [5] is one of the most popular distributed cluster-based routing protocols in wireless sensor networks. Each cluster head communicates using different CDMA codes in order/to in reduce interference from nodes belonging to other clusters.

\section{Measures suggested as improvement in cluster based} heterogeneous network:

A considerable amount of research have been done in this area and simulation results shows that by applying various energy control strategies ,considering different parameters, an effective results can be obtained.

A self organizing clustering algorithm CODA i.e. Cluster based self-Organizing Data Aggregation method based on the distance from the sink and an aggregating data using competitive machine learning [6].

CODA divides the whole network into a small number of groups based on the distance from the base station and the strategy of routing and each group has its own number of cluster members and member nodes.

EDGA algorithm to achieve good performance in terms of lifetime by minimizing energy consumption for in -network communications and balancing the energy load. It is based on weighted election probabilities of each node to become a cluster head, which can better handle the heterogeneous energy.

In CBRP(Clustered based routing hierarchal routing protocol , a new concept called headset, consist of one active cluster head and some other associate cluster heads with in the cluster [12].

The head set members are responsible for control and management of the network the head set is responsible to send message to the base station. results shows that this protocol performance better as compare to LEACH in context to energy consumption, frame transmission, and lifetime of the network.

RCFT(Re-clustering formation technique) suggested is to disperse and re-organise cluster heads considering number of hops between clusters organised randomly and the belonging nodes for the sake of the efficient division of clusters. This technique aims to elect cluster head efficiently which has a direct impact on energy consumption.

A HWSNs (Heterogeneous wireless sensor network model) based on energy and computational heterogeneity [19].EDFM is a self-adaptive clustering routing protocol similar with LEACH. The algorithm tries to balance energy consumption round by round, which will provide the longest stable period for the networks.

\section{Chain based approach}

The main idea in PEGASIS [14] is for each node to receive from and transmit to close neighbours and take turns being the leader for transmission to the base station. This approach will distribute the energy load evenly among the sensor nodes in the network.

We initially place the nodes randomly in the play field, and therefore, the $i$-th node is at a random location. The node will be organized to form a chain, which can either be accomplished by the sensor nodes themselves using a greedy of nodes. Each node that has elected itself cluster head for the current round broadcasts an advertisement message to the rest of the nodes in the network.

For gathering data in each round, each node receives data from one neighbor, fuses with its own data, and transmits to the other neighbor on the chain. Node co will pass its data towards node $\mathrm{c} 2$. After node $\mathrm{c} 2$ receives data from node $\mathrm{cl}$, it will pass the token to node $c 4$, and node $c 4$ will pass its data towards node $\mathrm{c} 2$.

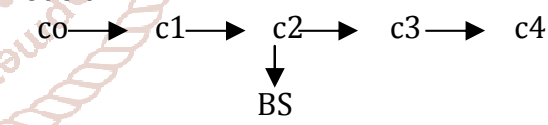

Fig4. Token passing approach

A considerable amount of research have been done in this area and simulation results shows that by applying various energy control strategies, considering different parameters, an effective results can be obtained.

In CBRP(Clustered based routing hierarchal routing protocol ,a new concept called headset, consist of one active cluster head and some other associate cluster heads with in the cluster [12]. The head set members are responsible for control and management of the network the head set is responsible to send message to the base station. results shows that this protocol performance better as compare to LEACH in context to energy consumption, frame transmission, and lifetime of the network.

\section{Randomized approach}

Energy aware random asynchronous wakeup (RAW-E) protocol [22], a novel cross layer power management and routing protocol for heterogeneous wireless sensor and actor networks, RAW-E is a distributed, randomized algorithm where nodes make local decision on whether to sleep or to be active based on the energy level of its neighbors. 


\section{ANALYSIS OF PROGRESSIVE WORK:}

An overview of protocols proposed for heterogeneous networks is given in the table 1 . These protocols need to be improved further or new protocols should be developed to address. We can extend these protocols to deal with more than three types of nodes and to include more than two level of hierarchy.

Important issues/factors that can be explored in these models where the heterogeneity among sensor nodes is not only in their available energy, but also in their processing capabilities and even in energy consumption in their data processing (compression, fusion) etc

Future work could explore similar issues in query driven and event driven types of sensor networks and even multi hop clustering and fault tolerant mechanism could be used in heterogeneous sensor networks.

\section{Cost analysis}

In this paper, heterogeneous nodes are classified as: Normal ('Nn'), Super ('Ns') and Advanced ('Na') nodes. All the three types of nodes are using the deterministic sensing model proposed by Ming et al [16] but the sensing range of super nodes is higher than that of advanced nodes. In this model, an event is detected if the strength of the received signal is within the sensing threshold set for event detection.

For communication model, first order radio model as proposed by Wendi et al [17] is used.

The default communication and the sensing range of ' $N n^{\prime}$, ' $N S$ ', and ' $N a$ ' are defined as 'Rcn', 'Rsn' and 'Rcs', 'Rss' and 'Rca', ' $R s a$ ' respectively. The underlying assumption for the communication and sensing range is 'Rca $>R c s>R c n$ ' and 'Rsa $>$ Rss $>$ Rsn'. The sensor node cost is determined by communication range and sensing range of sensor as suggested by Chun-Hsien Wu \& Yeh-Ching Chung [18].

This is evaluated as the extra cost of high power sensors per unit energy savings done by that particular level of the network.

Energysaving $=($ Energyinitial - Energyconsumed $),(6)$

where Energysaving is defined as the remaining energy in the network.

For 2-level Heterogeneous Model as proposed by Curt et al [19], Cost for sensor node deployment (D_Cost2-level) can be defined from deployment cost model as follows: $D_{-}$Cost2-level $={ }_{-} \operatorname{Num}(\mathrm{Ns}) * \mathrm{Ns}_{-} \cos t+\mathrm{Num}(\mathrm{Nn})_{-}$ Energysaving

$$
N s_{-} \operatorname{cost}={ }_{-} R c s+R 2 s s_{-} R c n+R 2 s n_{-}
$$

where $D_{-}$Cost2-level is evaluated as the total cost of deployed nodes per unit energy saving realized from the deployment of higher level nodes. Ns_cost is the difference of additional cost incurred by super nodes as compared to normal nodes. Cost factor as suggested by Duarte-Melo \& Mingyan [20] is defined by two factors only viz. communication range and sensing range represented by 'Rcs' and ' $R 2 s s$ ' respectively as rest all the parameters are assumed to be same.

For 3-level Heterogeneous Model, Deployment cost of sensor nodes can be derived from three types of nodes as follows:

$$
\begin{aligned}
& \text { D_Cost3-level } \left.=\_N u m(N a) * N a_{-} \text {cost }+ \text { Num(Ns }\right) * \\
& \text { Na_cost+Num(Ns)_Energysaving } \\
& N a_{-} \cos t=\_R c a+R 2 s a \_R c n+R 2 s n_{-}
\end{aligned}
$$

Where Na_cost is the diference between advanced nodes and normal nodes.

For n-level Heterogeneous model, ' $n$ ' random number of levels are defined. Hence, the deployment costs are derived as follows:

$$
\begin{gathered}
D \_C o s t n-l e v e l=\_i=n i=4 N u m(N i) * N i_{-} \operatorname{cost} \text { Energysaving } \\
N i \_c o s t=\_n a=1 \_R c a+R 2 s a_{-}+\_n s=1 \_R c s+R 2 s s \_n i=1 \_R c i+ \\
R 2 s I_{-}
\end{gathered}
$$

\section{CONCLUSION AND FUTURE WORK:}

In this article we have given a comprehensive survey of heterogeneous network in wireless sensor models. Throughout the paper efficient use of energy is given top priority. Various techniques under cluster based approach, chain based approach have been discussed to improve network life time, deployment cost, stability and throughput factors.

Comparison analyses of more heterogeneous protocols have been discussed in table 2 .

\section{REFERENCES:}

[1] Http://www.xbow.com/Products/Wireless_Sensor_Ne tworks.htm. Consultada el 1 de febrero de 2009.

[2] S. Muruganathan, D. Ma, R. Bhasin, A. Fapojuwo, "A Centralized Energy-Efficient Routing Protocol for Wireless Sensor Networks," IEEE Commun. Mag., 2005, Vol. 43, Issue 3, pp. 8-13.

[3] S. Olariu, Q. Xu "Information Assurance in Wireless Sensor Networks". Proceedings of the 19th IEEE International Parallel and Distributed Processing Symposium.2005. pp. 236-240.

[4] A. Mainwaring, J. Polastre, R. Szewczyk, D. Culler, J. Anderson. "Wireless Sensor Networks for Habitat Monitoring". Proceedings of the 1st ACM International workshop on wireless sensor Networks and applications. 2002. pp. 88-97.

[5] ZIgBee Specification. "ZigBee Document 053474r06, version 1.0". December 2004. http://www.zigbee.org/ Consultada el 1 de febrero de 2009.

[6] M. Augusto M. Vieira, D. C. da Silva Junior. "Survey on Wireless Sensor Network Devices". Proceedings of the IEEE conference Emerging Technologies and Factory Automatization. Vol. 1. 2003. pp. 537-544.

[7] J. N. Al karaki, A. E. Kamal. "Routing Techniques in Wireless Sensor Networks: A survey". IEEE Wireless Communications. Vol. 11. 2004. pp. 6-28.

[8] V. Ramasubramanian and D. Mosse, "Bra: a bidirectional routing abstraction for asymmetric mobile ad hoc networks," IEEE/ACM Transaction on Networking (TON), vol. 16, no. 1, 2008, pp. 116-129.

[9] D. Tian and N. D. Georganas, "Energy efficient routing with guaranteed delivery in wireless sensor networks," IEEE Wireless Communications and Networking Conference (WCNC), Mar. 2003, pp. 1923-1929.

[10] M. D. Yarvis, N. Kushalnagar, H. Singh, A. Rangarajan, Y. Liu, and S. Singh, "Exploiting heterogeneity in sensor networks," IEEE INFOCOM, Mar. 2005, pp. 878-890. 